\title{
Erratum to: Asymptotic formulae for likelihood-based tests of new physics
}

\author{
Glen Cowan $^{1}$, Kyle Cranmer ${ }^{2}$, Eilam Gross ${ }^{3}$, Ofer Vitells ${ }^{3, a}$ \\ ${ }^{1}$ Physics Department, Royal Holloway, University of London, Egham, TW20 0EX, UK \\ ${ }^{2}$ Physics Department, New York University, New York, NY 10003, USA \\ ${ }^{3}$ Weizmann Institute of Science, Rehovot 76100, Israel
}

Received: 24 June 2013 / Published online: 20 July 2013

(C) Springer-Verlag Berlin Heidelberg and Società Italiana di Fisica 2013

Erratum to: Eur. Phys. J. C (2011) 71:1554

DOI 10.1140/epjc/s10052-011-1554-0

Equation (74) should read

$p_{s+b}=\int_{q_{\mathrm{obs}}}^{\infty} f(q \mid s+b) d q=1-\Phi\left(\frac{q_{\mathrm{obs}}+1 / \sigma_{s+b}^{2}}{2 / \sigma_{s+b}}\right)$

Equation (75) should read

$p_{b}=\int_{-\infty}^{q_{\mathrm{obs}}} f(q \mid b) d q=\Phi\left(\frac{q_{\mathrm{obs}}-1 / \sigma_{b}^{2}}{2 / \sigma_{b}}\right)$

Acknowledgement We thank Christian Gumpert for spotting the typographical error in the original paper.

The online version of the original article can be found under doi:10.1140/epjc/s10052-011-1554-0.

a e-mail: ofer.vitells@weizmann.ac.il 